\title{
Contents to Volume 116 (1999)
}

\section{Volume 116, No. 1, March 1999}

Claus Hertling: Classifying Spaces for Polarized Mixed Hodge Structures and for Brieskorn Lattices

Frazer Jarvis: Mazur's Principle for Totally Real Fields of Odd Degree

\section{Volume 116, No. 2, April 1999}

Shigeyuki Kondō: On the Kodaira Dimension of the Moduli Space of $K 3$ Surfaces II

Antonio Campillo, Janusz Grabowski and Gerd Müller: Derivation Algebras of Toric Varieties

Solomon Friedberg and David Goldberg: On Local Coefficients for Nongeneric Representations of Some Classical Groups

Vesselin Gasharov: Hilbert Functions and Homogeneous Generic Forms II

Donu Arapura and Madhav Nori: Solvable Fundamental Groups of Algebraic Varieties and Kähler Manifolds

Andreas Langer: Local Points of Motives in Semistable Reduction

Xiangjun Song and Thomas J. Tucker: Dirichlet's Theorem, Vojta's Inequality, and Vojta's Conjecture

Errata: Barry Green and Michel Matignon: Lifting of galois covers of smooth curves, Compositio Math. 113 (1998), 237-272

Volume 116, No. 3, May (I) 1999

Yves Laurent: Vanishing Cycles of Irregular $\mathcal{D}$-Modules 